\title{
Management of Inguinal Hernia in a Tertiary Center, a two-year Retrospective Study.
}

\author{
Dritan Çobani ${ }^{1}$, Agron Dogjani ${ }^{2 *}$, Arben Gjata ${ }^{2}$, Kastriot Haxhirexha ${ }^{3}$, Hysni Bendo ${ }^{4}$
}

Received: 05 May 2021 / Accepted: 01 June 2021 / Published online: 20 July 2021

This article is published with open access at https://journal.astes.org.al

(C) The author(s) 2021. \& Copyright (C) 2021, the Albanian Society for Trauma and Emergency Surgery

(c) The Albanian Journal of Trauma and Emergency Surgery is an Open Access Journal. All articles are distributed under the terms of the Creative Commons Attribution Non-Commercial License: http://creativecommons.org/licenses/by-nc/4.0/) which permits unrestricted non-commercial use, distribution, and reproduction in any medium provided the original work is properly cited.

\section{Abstract}

Background: Inguinal hernia repair remains the most common surgery performed by general surgeons worldwide. There is a lot of published data on the surgical management of inguinal hernias in our country. This study aims to describe our experiences in the surgical management of inguinal hernias and compare our results with those reported in the literature.

Material and Method: A descriptive retrospective study was conducted at the University Hospital of Trauma in Albania.

From April 2016 - March 2018 were recorded all the data for patients who presented to our hospital and underwent inguinal hernia repair in our hospital, under the conditions of elective surgery.

Statistical data analysis was done using SPSS software version 17.0.

Results: In this period had a total of 542 patients with inguinal hernias were enrolled in the study. The median age of patients was 46,12 years (range 14 to 92 years old).

Males outnumbered females by a ratio of $18.3: 1$. This gender difference was statistically significant $(\mathrm{p}=0.004)$. Most patients 130 (23.9\%) presented late (more than one year of onset of hernia).

At presentation, 208 (38.4\%) patients had a reducible hernia, 101(18.6\%) had an irreducible hernia. The majority of patients $303(55.9$ $\%)$ had a right-sided inguinal hernia, and 156(28.7\%) had left-sided inguinal hernia with a right-to-left ratio of 1.94: 1. Eighty-three $83(15.3 \%)$ patients had bilateral inguinal hernias. 319 (58.8\%) patients had an indirect hernia,

Conclusion: Inguinal hernias continue to be a source of morbidity and mortality in our center. Early presentation and elective repair of inguinal hernias is pivotal in order to eliminate the morbidity and mortality associated with this very common problem.

Keywords: Inguinal hernias, Surgical management, Treatment outcome, Predictors of outcome.

\section{Introduction}

The inguinal hernia is one of the oldest recorded maladies of suffering with references on ancient Egyptian papyrus dating back to $1552 \mathrm{BC}[1]$. The earliest herniorrhaphy was performed on Pharaoh Merneptah (1224 e1214 BC) [2].

Original article, no submission or publication in advance or in parallel

* Corresponding author:

Asc. Prof. Dr. Agron Dogjani MD, Ph.D. FACS, FISS, FICS

$\bowtie \quad$ agron.dogjani@umed.edu.al

1 General Surgeon at the University Hospital of Trauma, Tirana, ALBANIA.

2 University of Medicine of Tirana, ALBANIA.

3 The State University of Tetovo, Faculty of Medicine, R.N MACEDONIA

4 Resident Internal Medicine SRH Clinic Karlsbad, Karlsruhe GERMANY
The word "hernia" is derived from a Latin term meaning "a rupture." [3]. An inguinal hernia is a protrusion of the contents of the abdominal cavity or preperitoneal fat through a hernia defect in the inguinal area [4].

Inguinal hernia is one of the most common problems encountered by general surgeons and can have significant complications [5]. Globally, inguinal hernia is the most common type of hernia, comprising approximately $75 \%$ of all abdominal wall hernias [6]. Inguinal hernia repair is one of the most common general surgical operations worldwide accounting for 10 to $15 \%$ of all surgical procedures and is the second most common surgical procedure after appendectomy [7]. It is estimated that worldwide over 20 million inguinal hernia repairs are performed each year, specific levels of functioning vary between countries from about $100-300$ per 100,000 population per year [4]. In the United Kingdom, approximately 100,000 inguinal hernias 
are repaired each year, and approximately 750,000 inguinal hernias are repaired each year in the United States. However, less than $40 \%$ are actually repaired, resulting in many patients developing long-term inguinal hernia associated with a higher incidence of morbidity and mortality.[4]

Inguinal hernia is operated on to reduce symptoms when they are present with acute complications or to prevent complications.

Patients with symptomatic inguinal hernias force the symptoms to appear to the doctor and undergo the intervention, while patients with asymptomatic or even minimal symptoms present a great dilemma both for themselves and for the doctor regarding the attitude towards them.

In choosing a treatment tactic, all of the following factors must be taken into account; recurrence risk; risk of complications; postoperative recovery and quality of life (return to work); degree of difficulty and realization of the technique by the surgeon (learning curvature); costs (hospital and social costs)

Since Bassini published his original description of inguinal hernia repair in 1887, many techniques for hernia repair such as Shouldice, Darning, Desarda, Modified Bassini, Lichtenstein mesh repair [7, 8].

In the diagnostic protocols for inguinal hernia in adult patients we rely on these points as: a) Anamnesis that includes: Swelling in the groin, right / left, type of complaints (pain), their duration, the presence of swelling in the inguinal region, signs and symptoms of irreponibility, reduction of hernial mass, previous hernia surgeries;[10] b) Predisposing factors: smoking, chronic obstructive pulmonary disease (COPD), hard work, with lifting weights for a long time, positive family history, previous operations on the inferior hypochondrium.; c) Physical examination; herniated mass (reducible) (above the inguinal ligament), medial / lateral localization, the presence of scarring at the level of the inguinal region (evidence of a previous operation), evaluation of the contralateral hernial gate, symptoms of reproducibility, reducibility, test involvement and region scrotal, the presence of ascites, examination of the colon; [10] d) Differential diagnosis with all tumors of the inguinal region such as: Femoral hernia, incisional hernia, lymph node enlargement, aneurysm, saphenous varices, soft tissue tumors, abscesses, genital abnormalities (ectopic testis); [10] e) Presence of pain and differentiation from pain caused by: adductor tendon, pubic osteitis, osteoarthritis of the thigh, bursitis ileopectinea, radiation of lumbar pain.[10]

Examination of a patient with a suspected inguinal hernia should: first - check the patient when he / she is standing; secondly - the identification of the hernial mass through the cough process; the third performs abdominal examination with the patient lying down; fourth make the differentiation between direct and indirect hernia; fifth, make a differential diagnosis with: femoral hernia, hydrocele (differentiated from inguinal hernia), varicose veins ... [10]
The purpose of our study was: Evidence and evaluation of the results of surgical treatment of primary inguinal hernia treated in planned surgery according to two different surgical methods and techniques (open technique with and without mesh, respectively according to Lichtenstein and Bassini technique) in adults, for identify the treatment method that has the best efficacy in: Post-operative complications; the decrease of the hospital stay; decreased recidivism, late complications, and consequently lower mortality and morbidity.

All study variables of our study included; demographic data; Clinical evaluation before intervention (according to ASA) and correlation with postoperative complications and BMI; postoperative outcomes in herpes and massless hernioplasties in patients with primary inguinal hernia; Evidence of technical repair effects [(Bassini, Shouldice, Desarda technique) vs. Lichtenstein technique], and their correlation with ASA, gender and age, type of hernia; the effect of operative antibiotic-prophylaxis; assessment of postoperative hospital stay days; complications, morbidity, complications (early and late) and mortality.

\section{Study Methodology}

Our study is of the case-control type (based on the comparison of two randomized groups that undergo different surgical methods for the treatment of primary inguinal hernia), with two components: a) Descriptive: this component refers to the description / assessment of the prevalence of primary inguinal hernia by gender, age group, BMI, ASA, hernia localization, operative wound complications, complications (early and late) morbidity and mortality in planned surgery, in patients treated with and without mesh ; b) Analytical: refers to the assessment of the association of different variables with the Bassini, Shouldice, Desarda or Lichtenstein operative technique with operative wound complications, complications (early and late) morbidity and mortality, and which of these techniques has the most desired results postoperative.

Study time includes the period of a) surgical treatment From April 2016 - March 2018 and b) postoperative followup from April 2016 - March 2018.

Patients were followed on day 3, 5, 7 after surgery, one, three months and every year after surgery. The maximum attendance was 6 years and the minimum was 3 years.

The study included 542 patients hospitalized, diagnosed and treated at the University Hospital of Trauma Tirana, Albania.

In our study was recorded $429(79.3 \%)$ patients, who was treated with mesh (according to Lichtenstein) and 113 $(20.7 \%)$ patients, who was treated without mesh (according to Bassini, Shouldice, Desarda technique).

Excluded from our study: patients under 14 years, pregnant women, femoral hernia; recurrent hernia; complicated primary inguinal hernias. 
Statistical data analysis was done using SPSS software version 17.0 (SPSS, Inc, Chicago, IL). Data was summarized in form of proportions and frequent tables for categorical variables. Continuous variables were summarized using means, median, mode and standard deviation. P-values were computed for categorical variables using Chi-square $(\chi 2)$ test and Fisher's exact test depending on the size of the data set.

Independent student t-test was used for continuous variables. Multivariate logistic regression analysis was used to determine predictor variables that are associated with outcome. A p-value of less than 0.05 was considered to constitute a statistically significant difference.

\section{Results}

In our study included 542 patients, their ages ranging from 14 to 92 years with an average age of 46.12 years, and 514 $(94.84 \%)$ were male and $28(5.16 \%)$ were female with a male-female ratio of 18.3: 1. (Tab.1) This gender difference was statistically significant $(\mathrm{P}=0.004)$.

Table 1 - Distribution of Inguinal hernia based on gender.

\begin{tabular}{|l|l|l|l|l|}
\hline Gender & Frequency & \% & Valid \% & Cumulative \% \\
\hline Male & 514 & 94.84 & 94.84 & 94.84 \\
\hline Female & 28 & 5.16 & 5.16 & 100.0 \\
\hline Total & 542 & 100.0 & 100.0 & \\
\hline
\end{tabular}

At presentation, 208 (38.4\%) patients had reducible hernia, 101(18.6\%) had irreducible hernia. The majority of patients 303(55.9\%) had right sided inguinal hernia, and $156(28.7 \%)$ had left sided inguinal hernia with a right-toleft ratio of 1.94:1.

Eighty-three (15.4\%) of patients had bilateral inguinal hernias. (Tab.2) Three hundred and nineteen (58.8\%) of patients had indirect inguinal hernia, 176 (32.6\%)of patients had direct inguinal hernia, and $47(8.6 \%)$ had both indirect and directinguinal hernia (hernia pantaloon).

Table 2 - Distribution of Inguinal hernia based on localization.

\begin{tabular}{|l|l|l|l|l|}
\hline Inguinal hernia & Frequency & \% & Valid \% & Cumulative \% \\
\hline Left side & 156 & 28.7 & 28.7 & 28.7 \\
\hline Right side & 303 & 55.9 & 55.9 & 84.6 \\
\hline Bilateral & 83 & 15.3 & 15.4 & 100.0 \\
\hline Total & 542 & 100.0 & 100.0 & \\
\hline
\end{tabular}

History of heavy weight lifting (construction worker, unloading load) was found in 111 (20.4\%) patients, and 227 (41.8\%) patients were smokers.

The time of clinical presentation of inguinal hernia disease (the time of onset of complaints until surgery) ranged from 1 day to 45 years. Most patients 130 (23.9\%) presented late (more than one year of onset of hernia).
Concomitant diseases were encountered in $124(22.8 \%)$ patients. Of these, $30(24.1 \%)$ had chronic lung infections, $39(31.4 \%)$ had hypertension, $27(21.7 \%)$ had diabetes mellitus, and $28(22.8 \%)$ patients had cardiovascular diseases. (Tab. 3)

Table 3 - Distribution of concomitant Diseases

\begin{tabular}{|l|l|l|l|l|}
\hline Concomitant Diseases & Frequency & \% & Valid \% & Cumulative \% \\
\hline COPD & 30 & 24.1 & 24.1 & 24.1 \\
\hline Hypertension & 39 & 31.4 & 31.4 & 55.5 \\
\hline Diabetes Mellitus & 27 & 21.7 & 21.7 & 77.2 \\
\hline Cardiovascular Diseases & 28 & 22.8 & 22.8 & 100.0 \\
\hline Total & 124 & 100.0 & 100.0 & \\
\hline
\end{tabular}

All of these concomitant medical conditions were checked before surgery.

According to the ASA classification, 317(58.4\%) patients had class I ASA; $113(20.8 \%)$ had class II ASA, $81(14.9 \%)$ had ASA class III, $22(2.9 \%)$ had ASA class IV and $10(2.0 \%)$ patients had ASA class V. All patients in this study underwent open herniorrhaphy/ hernioplasty. (Tab. 4)

Table 4 - Distribution of patients with Inguinal hernia based on ASA classification

\begin{tabular}{|l|l|l|l|l|}
\hline ASA classification & Frequency & \% & Valid \% & Cumulative \% \\
\hline Class I & 317 & 58.4 & 58.4 & 58.4 \\
\hline Class II & 113 & 20.8 & 20.8 & 79.2 \\
\hline Class III & 81 & 14.9 & 14.9 & 94.1 \\
\hline Class IV & 21 & 3.9 & 3.9 & 98.0 \\
\hline Class V & 10 & 2.0 & 2.0 & 100.0 \\
\hline Total & 542 & 100.0 & 100.0 & \\
\hline
\end{tabular}

Elective hernia surgery was performed in all patients, which was performed under spinal anesthesia in 286 (52.7\%) patients, general anesthesia in $141(26.1 \%)$ patients and local anesthesia in 115 (21.2\%) patients. (Tab. 5)

Table 5 - Distribution of patients with Inguinal hernia based on sort of anesthesia

\begin{tabular}{|l|l|l|l|l|}
\hline Anesthesia & Frequency & \% & Valid \% & Cumulative \% \\
\hline Spinal & 286 & 52.7 & 52.7 & 52.7 \\
\hline General & 141 & 26.1 & 26.1 & 78.8 \\
\hline Local & 115 & 21.2 & 21.2 & 100.0 \\
\hline Total & 542 & 100.0 & 100.0 & \\
\hline
\end{tabular}

Posterior inguinal canal wall repair was used by Bassini modified technique repair in $36(6.6 \%)$ patients, Shouldice technique repair in $51(9.4 \%)$, and Desarda technique repair in $26(4.7 \%)$ patients, respectively. Four hundred and twenty-nine (79.3\%) patients had repair of hernias according to Lichtenstein technique. (Tab. 6) 
Table 6 - Distribution of patients according the of technique of herniorrhaphy.

\begin{tabular}{|l|l|l|l|l|}
\hline $\begin{array}{l}\text { Technique of } \\
\text { Herniorrhaphy }\end{array}$ & Frequency & $\%$ & Valid \% & Cumulative \% \\
\hline Bassini & 36 & 6.6 & 6.6 & 6.6 \\
\hline Shouldice & 51 & 9.4 & 9.4 & 16.0 \\
\hline Desarda & 26 & 4.7 & 4.7 & 20.7 \\
\hline Lichtenstein & 429 & 79.3 & 79.3 & 100.0 \\
\hline Total & 542 & 100.0 & 100.0 & \\
\hline
\end{tabular}

Outcome of surgical treatment; In this study, a total of 62 postoperative complications were recorded in 60 $(11 \%)$ patients, of these, surgical site infection was the most common postoperative complication, accounting for 26 $(43.3 \%)$ of cases.

All complications were resolved only with conservative treatment. Duration of hospital stay (LOS) ranged from 1 to 7 days. The length of hospital stay was significantly longer in patients with advanced age, concomitant medical illness, upper ASA classification.

In this study, four patients died giving a mortality rate of $0.7 \%$. Longer duration of symptoms, late hospitalization, cohabiting illness, high ASA grade, and the presence of complications were found to be predictors of mortality ( $\mathrm{P}$ $<0.001)$.

Patients were available for follow-up at 3 months, 6 months and 1 year, respectively.

The average duration of follow-up after surgery was eight months (1-24 months).

Chronic groin pain was recorded in 48 (8.8\%) of cases, most of which improved in minor analgesics. There were only seven $(1.2 \%)$ cases of hernia recurrences (5 post-Bassini, 1 post- Desarda, 1 post-Shouldice, 1 postLichtenstein repairs).

\section{Discussion}

Inguinal hernia repair is already a common and routine practice for every surgeon and at the same time occupies an important part of the surgical activity and in terms of frequency ranks immediately after appendectomy.[13]

In our study included 542 patients, their ages ranging from 14 to 92 years with an average age of 46.12 years, and $514(94.84 \%)$ were male and $28(5.16 \%)$ were female with a male-female ratio of 18.3 : 1 , and was found to be statistically significant. $(\mathrm{p}<0.00001)$.

Charles et al reported $93.2 \%$ of all the cases to be males.[14] Also Russell et al. found Inguinal hernias are quoted as being 20 times more common in men than women [15]. Our results are similar, in fact showing that inguinal hernia repairs were carried out in total almost 18.3 times more commonly in men than women. Velanovich et al. found a prevalence of hernia among $85 \%$ of males and $15 \%$ of females [16], Osifo et al. has cited; the median age of 46 years was similar [17].
The possible reason of high preponderance of inguinal hernia in males is their more involvement in strenuous activities. Inguinal hernia has a life time prevalence of $27 \%$ in men and $3 \%$ in women [1], which appears to drop after 45 years of age [8].

At our study we have recorded, 208 (38.4\%) patients had reducible hernia, 101(18.6\%) had irreducible hernia. The majority of patients $303(55.9 \%)$ had right sided inguinal hernia, and 156(28.7\%) had left sided inguinal hernia with a right-to-left ratio of 1.94: 1. Russell et al. found Inguinal hernias are also quoted to be right-sided in $55 \%$ of cases. [15] Our results have mirrored this slight right predominance, with $28.7 \%$ left and $55.9 \%$ right-sided repairs.

Charles et al. also reported right sided hernias to be more prominent than the left side.[14]

Eighty-three $83(15.3 \%)$ patients had bilateral inguinal hernias. Three hundred and nineteen $(58.8 \%)$ patients had indirect hernia, and $47(8.6 \%)$ had both indirect and direct types (hernia pantaloon).

The risk of inguinal hernia increases with age, and the annual incidence is around 50 percent by the age of 75.[18]

Approximately two-thirds of inguinal hernias are indirect, and one-third are direct.[19] Approximately 10 percent of cases are bilateral. [20] As in adults, about 10 percent of cases involve bilateral hernia.[8]

History of heavy weight lifting (construction worker, unloading load) was found in $111(20.4 \%)$ patients, and 227 $(41.8 \%)$ patients were smokers.

The time of clinical presentation of inguinal hernia disease (the time of onset of complaints until surgery) ranged from 1 day to 45 years. Most patients 130 (23.9\%) presented late (more than one year of onset of hernia).

According Bronsther et al. The incidence of $\mathrm{IH}$ has been reported as high as $4.4 \%$ and typically present as an asymptomatic bulge in the groin [21]

The hernia occurrence depends upon risk factors, and in our study the associated disease was encountered in 124 $(22.8 \%)$ patients. Of these, 30 (34.1\%) had chronic lung infections, 39 (31.4\%) had hypertension, 27 (21.7\%) had diabetes mellitus, and $13(10.4 \%)$ patients had cardiovascular disorders. Lau et al. showed the same thing in their article as below the hernia occurrence depends upon risk factors, including gender, age, complaints of cough, repeated pregnancies, constipation, prior surgeries, lifting of heavy weights, obesity, genetic predisposition, and smoking [22]

Lichtenstein repair has long been considered the 'gold standard' in inguinal hernia surgery [12, 23

Resolution of inguinal hernias are the most common procedures performed by surgeons and are an important factor in caring for the health of patients. associated with significant morbidity and mortality. There is no consensus on one type of intervention to address inguinal hernias. the type of procedure and the socioeconomic aspect. There is no adequate analysis for naturally untreated inguinal hernias. Many surgeons suggest that inguinal hernias be operated on and diagnosed in time. Incarcerated hernias and strangulations are conditions that must be treated. 


\section{Conclusion}

Elective hernia resolution is still one of the most common surgical procedures.

The decision for this operation stems from the research for the best comfort of the patients, from using a wellaccepted surgical technique, easy and fast to perform.

However, we believe that one operation is not needed to properly treat all types of hernias, when inguinal hernia is diagnosed, even in asymptomatic cases they should be offered repair as a necessary and mandatory final treatment.

Repair of inguinal hernia with nets is associated with lower rates of hernia recurrence.

Early complications include bruising, numbness, and wound infection.

Chronic pain is the predominant late complication.

COI Statement: This paper has not been submitted in parallel.

This research received no specific grant from any funding agency in the public, commercial, or nonprofit sectors. There are no relevant or minor financial relationships from authors, their relatives or next of kin with external companies. interest

Conflict of Interest Statement; There is no conflict of

Authorship Contribution; The first author has done the conception or design of the work and data collection and analysis. The second author has done the data analysis and interpretation as well as drafting the article and critical revision of the article. The third author did the English writing of the work, the collection, the data control, the English writing of the Article.

\section{References}

1. Lau WY. History of treatment of groin hernia. World J Surg 2002; 26: 748e59.

2. Thorwald J. The triumph of surgery. New York, NY: Pantheon; 1960. p. p 278.

3. Maingot's Abdominal Operations 12th Edition; Michael Zinner, MD and Stanley Ashley, MD (Eds) McGraw Hill Publishers, page. 127, 2013

4. Simons MP, Aufenacker T, Bay-Nielsen M, et al. European Hernia Society Guidelines on the Treatment of Inguinal Hernia in Adult Patients. Hernia. 2009; 13:343-403. 2.

5. Williams NS, Bulstrode CJK, O'Connell PR: Bailey \& Love's Short Practice of Surgery. 25th edition.London: Hodder Arnold; 2008.

6. Primatesta P, Golacre MJ: Inguinal hernia repair, incidence of electiveand emergency surgery. Int J Epidemol 1996, 25:835839.

7. Schools IG, Van Dijkman B, Butzelaar RM, Van Geldere D, Simons MP: Inguinalhernia repair in Amsterdam region. Hernia 2001, 5(1):37-40.
8. Kingsnorth AN, LeBlanc KA: Management of abdominal hernias. 3rd edition. London,New York: Edward Arnold; 2003:40-47.

9. Simons MP, Aufenacker T, Bay-Nielsen M, et al. European Hernia Society Guidelines on the Treatment of Inguinal Hernia in Adult Patients. Hernia. 2009; 13:343-403. 2.

10. Read RC. Herniology: Past, Present and Future. Hernia. 2009; 13: $577-80$.

11. Review Hernias: inguinal and incisional. Kingsnorth A, LeBlanc K Lancet. 2003 Nov 8; 362(9395):1561-71. [PubMed] [Ref list]

12. ARSLANI, N. (2020). Clinical Comparation of Postoperative Complications after Lichtenstein versus Bassini Inguinal Hernia Repair. Albanian Journal of Trauma and Emergency Surgery, 4(2), 695-698. https://doi.org/10.32391/ajtes. v4i2.118

13. E. Von Elm, D.G. Altman, M. Egger, S.J. Pocock, P.C. Gøtzsche, J.P. Vandenbroucke, Strobe initiative. The strengthening the reporting of observational studies in epidemiology (STROBE) statement: guidelines for reporting observational studies, Prev. Med. 45 (4) (2007 Oct 31) $247 \mathrm{e} 251$.

14. Charles NR. A two-year retrospective study of congenital inguinal hernia at western regional hospital, Nepal. J Nep Med Assoc. 2000;39:172-5.

15. Russell RCG, Williams NS, Bulstrode CJK Bailey \& Love's Short Practice of Surgery. 23rd Edition London: Hodder Arnold, 2000 [Google Scholar]

16. Velanovich, V., Shadduck, P., Khaitan, L. et al. Analysis of the SAGES Outcomes Initiative groin hernia database. Surg Endosc 20, 191-198 (2006). https://doi.org/10.1007/s00464005-0436-7

17. Osifo OD, Amusan TI: Outcome of giant inguinoscrotal hernia repair with local lidocaine anaesthesia. Saudi Med J. 2010, 31 (1): 53-58.

18. Nicks BA, Askew K. Hernias. eMedicine Web site. 2010 Jan 25. Available at: https://emedicine.medscape.com/ article/189563-overview. Accessed July 14, 2010.

19. Hammoud M, Gerken J. Inguinal Hernia. [Updated 2020 Sep 8]. In: StatPearls [Internet]. Treasure Island (FL): StatPearls Publishing; 2021 Jan-. Available from: https://www.ncbi.nlm. nih.gov/books/NBK513332/

20. Schneider E. Inguinal hernia: excerpt from the 5-minute Pediatric Consult. WD Web site. Accessed January 26, 2011.

21. Bronsther B, Abrahams MW, Elbonim C. Inguinal hernia in children-a study of 1000 cases and a review of the literature. J Am Med Womens Assoc 1972; 27: 5222e35

22. Lau H, Fang C, Yuen WK, Patil NG: Risk factors for inguinal hernia in adult males: a case-control study. Surgery. 2007, 141:262-266. 10.1016/j.surg.2006.04.014

23. Anadol AZ, Akin M, Kurukahvecioglu O, Tezel E, Ersoy E. A prospective comparative study of the efficacy of conventional Lichtenstein versus self-adhesive mesh repair for inguinal hernia. Surg Today. 2011 Nov;41(11):1498-503. doi: 10.1007/ s00595-011-4545-8. Epub 2011 Oct 4. PMID: 21969152 